IX International Scientific Symposium

"Farm Machinery and Processes Management in Sustainable Agriculture", Lublin, Poland, 2017

DOI: $10.24326 /$ fmpmsa.2017.10

\title{
CLOTHOID AS A TRANSITION CURVE OF THE MANIPULATOR END-EFFECTOR TRAJECTORY FOR HARVESTING TOMATOES IN A GREENHOUSE
}

\author{
Marek BORYGA, Pawel KOLODZIEJ, Krzysztof GOŁACKI \\ University of Life Sciences in Lublin, POLAND \\ E-mail of corresponding author: marek.boryga@up.lublin.pl
}

Keywords: precision harvest, trajectory planning, clothoid, tomatoes, greenhouses,

\begin{abstract}
The article presents the way of using the clothoid as a transition curve when planning the trajectory of the tomato manipulator end-effector during harvesting in a greenhouse. Three variants of end-effector motion trajectory were planned. The first path consists of two rectilinear segments connected by a circular arc. The second path is composed of a rectilinear segment - clothoid - circular arc - clothoid and a rectilinear segment. The third option consists of two rectilinear segments connected by a biclothoid. For the analysed variants of the manipulator gripper trajectories, the motion parameters were determined and compared, and the results were presented in the graphical form. The algorithm with the use of clothoid, proposed in the paper, ensures the continuity of the displacement, velocity and acceleration for the planned trajectory of the end-effector. This is particularly important for the dynamics of the manipulator arm movement.
\end{abstract}

\section{INTRODUCTION}

The use of robots in greenhouses and orchards, for example for fruit or vegetable harvesting and spraying applications is an important issue for precision agriculture. The first stage of designing technological operations using agricultural manipulators is planning the motion trajectory. The issue concerns two questions: determination of the trajectory of aggregates or mobile robots movement in the area plane as well as that of the end-effector towards a chosen object e. g. a fruit, a vegetable or a stem etc. The paper by Sabelhaus et al. (2013) presents the way of determining the trajectory of recurrent turns whose main construction element is a clothoid. The authors analysed seven different manoeuvres useful from the agronomic point of view and proved that all motions can be executed using the proposed method. Wilde (2009) presented a simple and fast method for calculation of sharpness and curvature of the clothoid for the trajectory of continuous profile. He used the fragments of clothoid to combine rectilinear segments with the circular arc. The algorithm generates smooth, natural, drivable paths using a minimal amount of steering to reach a desired end position. In the case of planning the arm motion, Baur et al. (2013) proposed two approaches to automation determination of the endeffector, trajectory in the working space of the manipulator - heuristic as well as that exploiting an artificial function of the potential. The experimental results are presented and discussed based on harvesting one fruit and one stem. Schuez et al. (2015) proposed a two-stage solution of trajectory planning the redundant manipulator. Motion trajectories are established using the reverse algorithm of kinematics and optimized by the direct method. The paper by Zhang et al. (2016) presents the optimized structure of manipulator with four degrees of freedom. Moreover, the authors stated that the planned cycloidal trajectory of the end-effector motion allows to avoid collision with the obstacle such as a stem, a leaf or other fruit etc. In the paper by Boryga and Graboś (Graboś and Boryga 2013, Boryga 2014) the authors used higher degree polynomials for planning manipulators motion trajectories. Their advantage is the zero value of jerk in the initial and final motion phases which is decisive, any others, for positioning accuracy, dynamic load of driving units and steering the arm motion. In addition, in the paper by Boryga et al. (2015) there was applied the PR-APT (Planning Rectilinear-Arc Polynomial 
Trajectory) method for planning the end-effector motion trajectory during harvesting tomatoes in a greenhouse. This paper presents the way of using the clothoid as a transient curve in planning the manipulator end-effector motion trajectory for harvesting tomatoes in a greenhouse. Three variants of end-effector motion trajectory were planned. The first path was composed of two rectilinear segments combined with the circular arc. The second was a rectilinear segment-clothoid-circular arc-clothoid and a rectilinear segment. The third path includes two rectilinear segments combined with the biclothoid (Kobryn 2017). The end-effector motion trajectories defined in this way can constitute partly fragments of obstacle profile to be avoided e. g. tomatoes stem with unripe fruit. For the analysed variants of the manipulator end-effector trajectory the motion parameters courses were determined and compared. Their results are presented graphically.

\section{PLANNING OF MOTION TRAJECTORY}

\section{Assumptions}

For all trajectories acceleration on the rectilinear segments is described by the $9^{\text {th }}$ degree polynomial (Boryga 2015):

$$
a(t)=-p_{252} \cdot t^{2} \cdot\left(t-0.5 t_{e}\right)^{5} \cdot\left(t-t_{e}\right)^{2}
$$

where: $p_{252}$ - the polynomial coefficient, $t_{e}$ - the time of motion end.

On the segment $B T_{1}$ the end-effector moves with the positive acceleration (the first part of the polynomial for which $0 \leq t<0.5 t_{e}$ ), however, for the segment $T_{2} E$ with negative acceleration (the second part of the polynomial for which $0.5 t_{e}<t \leq t_{e}$ ).
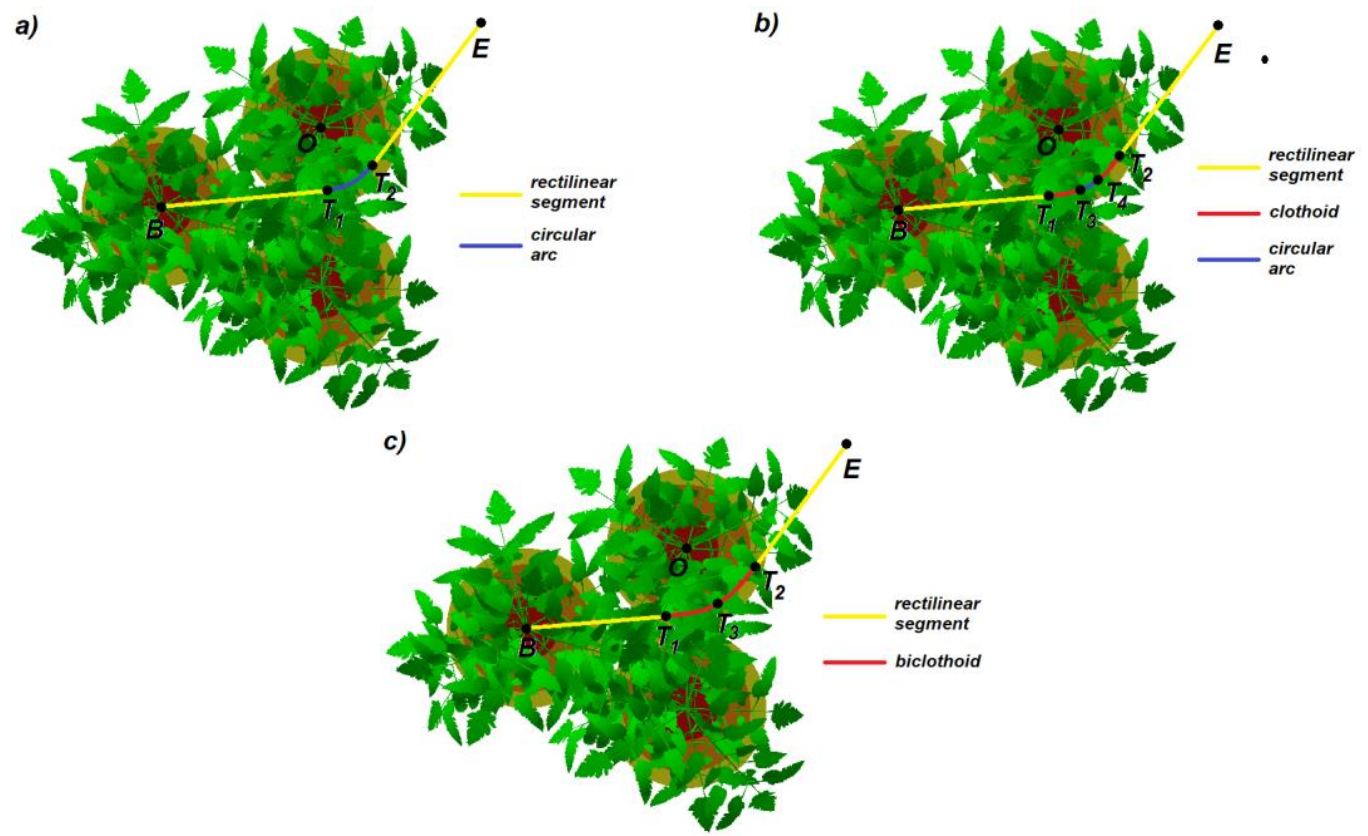

Fig. 1. Planned motion trajectories: a) Path 1 (rectilinear segment - circular arc - rectilinear segment), b) Path 2 (rectilinear segment - input clothoid - circular arc - output clothoid - rectilinear segment),

c) Path 3 (rectilinear segment - biclothoid - rectilinear segment)

For all trajectories, the constant variables are:

- The initial point $B$ and final point $E$ coordinates of the trajectory.

- The arc midpoint $O$ and arc radius $R$ coordinates constituting a fragment of obstacle outline to be avoided. 
- The global coordinates system $x y z$ whose origin is in the initial point of trajectory.

- The local coordinates system $x_{O} y_{O} z_{O}$ whose axes are parallel to those of the system $x y z$ but the origin is in the point $O$.

- The maximum velocity on the rectilinear segments and the steady velocity of the endeffector on the curvilinear fragments of trajectories $-v_{\max }$.

\section{Calculations for the clothoid segments}

The clothoid is a curve whose curvature $\kappa$ is proportional to the arc length $L$, expressed by the equations:

$$
L=A^{2} \kappa=\frac{A^{2}}{R} \quad \text { and } \quad \tau=\frac{L^{2}}{2 A^{2}}=\frac{L}{2 R}
$$

where: $A^{2}$ - the proportionality coefficient (clothoid parameter), $\tau$ - arc tangent direction.
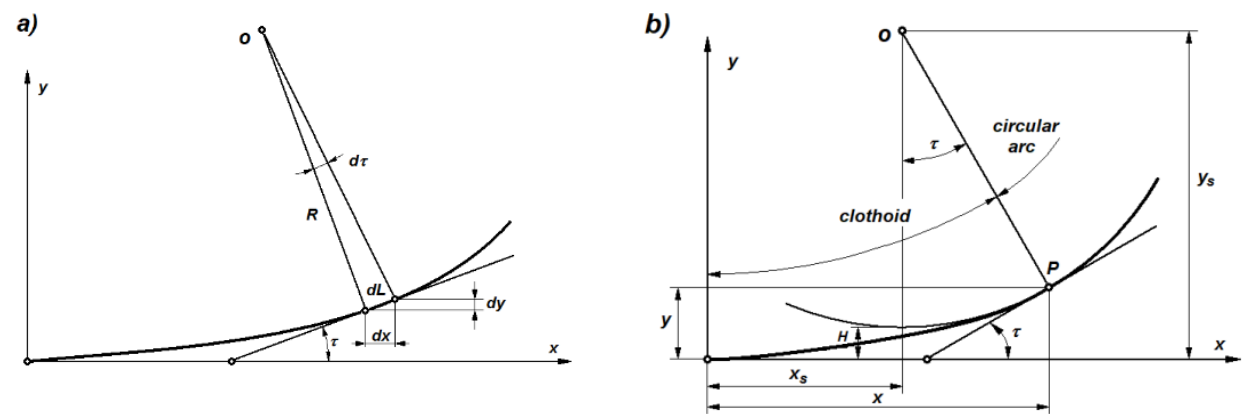

Fig. 2. Construction of an elementary clothoid: a) elementary triangle of clothoid, b) clothoid as a transition curve between rectilinear segments and circular arc

Rectangular coordinates of clothoid (Koc 2015):

$$
x=\int_{0}^{L} \cos \frac{L^{2}}{2 \cdot A^{2}} \cdot d L \quad y=\int_{0}^{L} \sin \frac{L^{2}}{2 \cdot A^{2}} \cdot d L
$$

The sequence of steps during calculation procedure of the clothoid segments is as follows: Step 1. The assumption (Path 2) or determination (Path 3) of clothoid length as well as calculation of curvature circle distant $H$ stand-off from the main tangent, time in the motion along the input and output clothoid and clothoid parameter.

Step 2. Introduction of the coordinate system $x_{T 1} y_{T 1} z_{T 1}$ whose axis $x_{T 1}$ is the direction of the motion on the segment $B T_{1}$ and its origin is in point $T_{1}$ as well as the system $x_{T 2} y_{T 2} z_{T 2}$ whose axis $x_{T 2}$ is the direction of the motion on the segment $T_{2} E$ and the origin is in point $T_{2}$.

Step 3. Determination of shifts in time for the motion along the clothoids.

Step 4. Determination of coordinates of the position of the end-effector in motion along: the input clothoid in the system $x_{T 1} y_{T 1} z_{T 1}$, the output clothoid in the system $x_{T 2} y_{T 2} z_{T 2}$, the input and output clothoid in the global coordinate system $x y z$.

\section{Calculation for rectilinear segments}

Step 1. Determination of coordinates of tangency points: $T_{1}, T_{2}$ of straight lines crossing points $B$ and $E$, respectively as well as tangents to the arc of midpoint in $O$ and the radius $R+H$ (for the Path $1 H=0$ ).

Step 2. Calculation of way increment on the rectilinear segments $B T_{1}$ and $T_{2} E$ denoted $\Delta s^{B T 1}$ and $\Delta s^{T 2 E}$.

Step 3. Determination of motion time on the segments $B T_{1}$ and $T_{2} E$. 


$$
t=\frac{\alpha \cdot \Delta s}{\beta \cdot v_{\max }}
$$

whereby $\alpha=1 / 61440, \beta=1 / 88704$ for the segment $B T_{1}$ it is assumed: $\Delta s=\Delta s^{B T 1}$, $t=t^{B T 1}$ however, for the segment $T_{2} E: \Delta s=\Delta s^{T 2 E}, t=t^{T 2 E}$.

Step 4. Determination of polynomial coefficient describing the acceleration course on the $B T_{1}$ and $T_{2} E$ segments:

$$
p_{252}=\frac{\beta^{10} \cdot v_{\max }^{11}}{\alpha^{11} \cdot(\Delta s)^{10}}
$$

For $B T_{1}: \Delta s=2 \cdot \Delta s^{B T 1}$ and $p_{252}=p_{252}^{B T 1}$, for $T_{2} E: \Delta s=2 \cdot \Delta s^{T 2 E}$ and $p_{252}=p_{252}^{T 2 E}$.

Step 5. Determination of the time shift for the motion on the $T_{2} E$ segment and coordinates of position of the end-effector in motion along the rectilinear segments.

\section{Calculations for the arc}

Step 1. Determination of time of motion along the arc, dependences for calculation of the angle displacement in the local coordinate system $x_{o} y_{O} z_{O}$ and determination of the coordinates of the end-effector position during the motion along the arc in the global coordinate system $x y z$.

Step 2. Determination of time shift for the motion along the arc.

\section{Final calculations}

Step 1. Determination of total end-effector dislocation along the trajectory and determination of first and second derivatives of dislocation in relations to time.

Step 2. Determination of total time of motion along the trajectory.

\section{RESULTS OF THE SIMULATION}

The path presented in Fig. 3 as a result of simulation refer to the motion in the plane $x y$.

a)

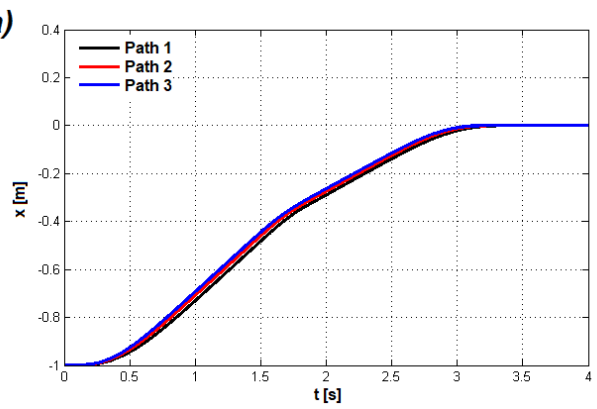

c)

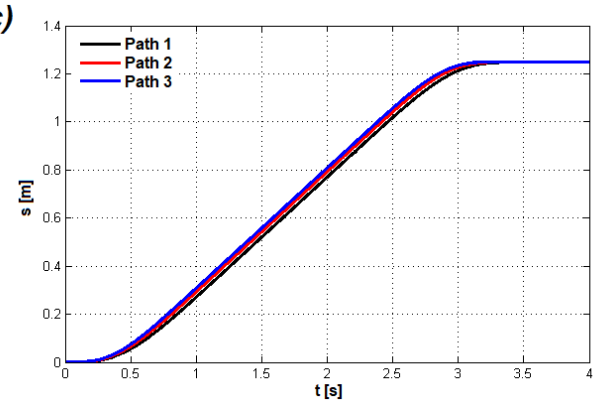

b)

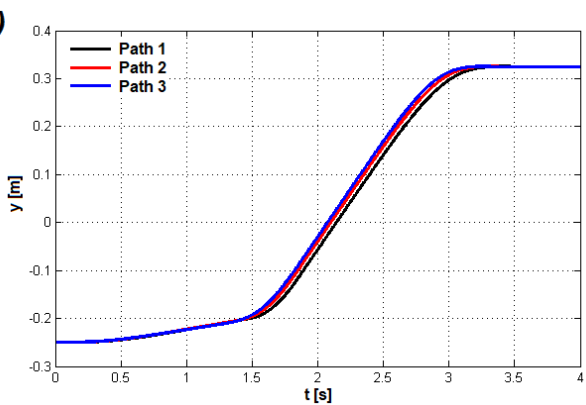

d)

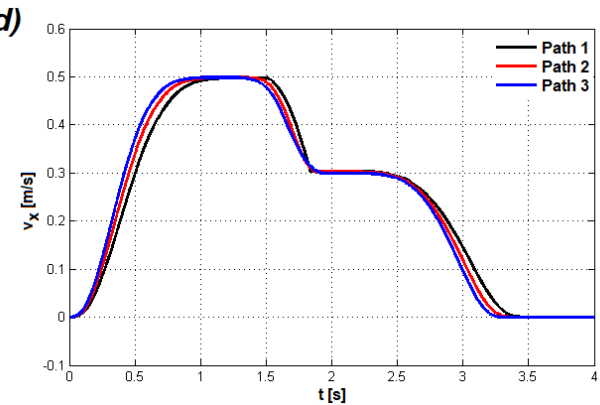


e)

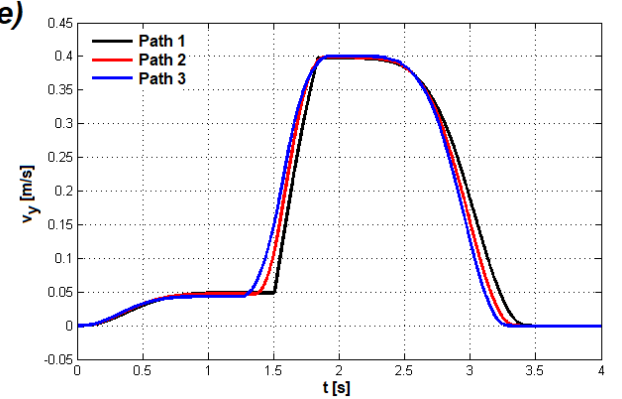

g)

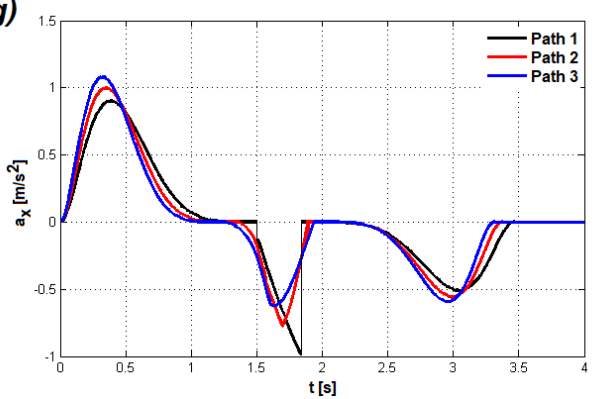

f)

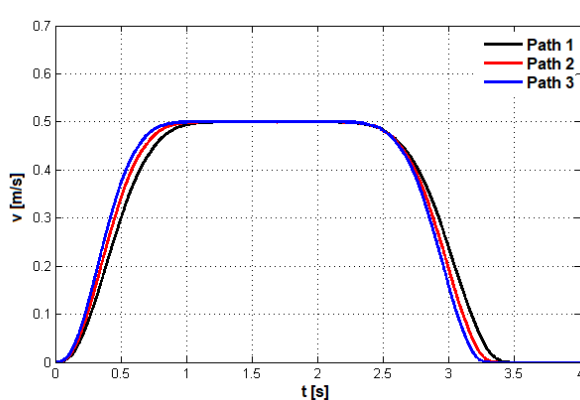

h)

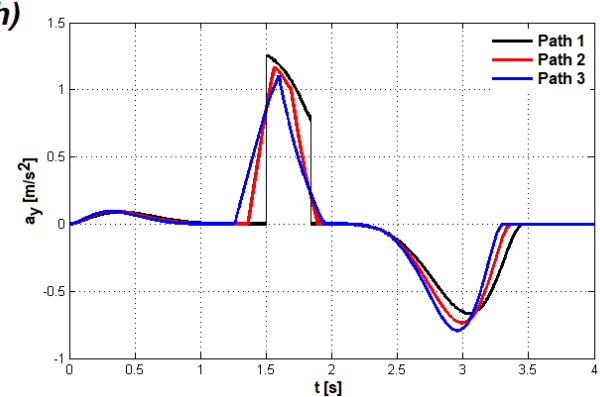

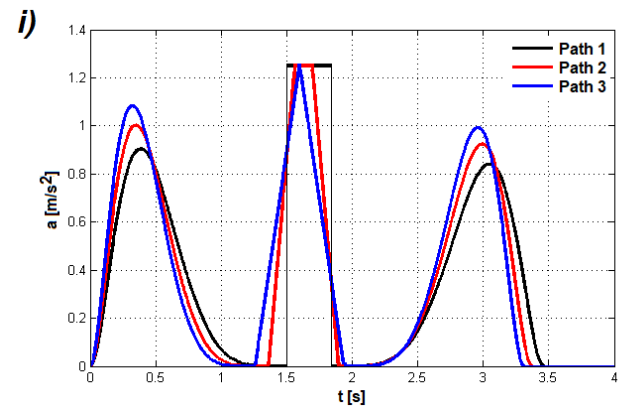

Fig. 3. The courses of kinematic quantities of the end-effector: a) dislocation towards the axis $x$, b) dislocation towards the axis $y, \mathrm{c})$ resultant dislocation, d) velocity oriented towards the axis $x$, e) velocity oriented towards the axis $y, \mathrm{f}$ ) resultant velocity, g) acceleration oriented towards the axis $x$, h) acceleration oriented towards the axis $y$, i) resultant acceleration.

Table 1. Comparison of the length of path and time of the motion for individual trajectories

\begin{tabular}{|c|c|c|c|}
\hline Path denotation & Construction of the path & Length $[\mathrm{m}]$ & Motion time $[\mathrm{s}]$ \\
\hline \multirow{4}{*}{ Path 1} & Rectilinear segment - $B T_{l}$ & 0.522015 & 1.507 \\
\hline & Circular arc $-T_{1} T_{2}$ & 0.164122 & 0.328 \\
\hline & Rectilinear segment $-T_{2} E$ & 0.561805 & 1.622 \\
\hline & 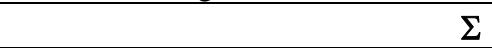 & 1.247942 & 3.457 \\
\hline \multirow{6}{*}{ Path 2} & Rectilinear segment - $B T_{l}$ & 0.471318 & 1.361 \\
\hline & Input clothoid $-T_{1} T_{3}$ & 0.1 & 0.2 \\
\hline & Circular arc $-T_{3} T_{4}$ & 0.065660 & 0.131 \\
\hline & Output clothoid $-T_{4} T_{2}$ & 0.1 & 0.2 \\
\hline & Rectilinear segment $-T_{2} E$ & 0.511165 & 1.476 \\
\hline & $\Sigma$ & 1.248143 & 3.368 \\
\hline \multirow{5}{*}{ Path 3} & Rectilinear segment - $B T_{1}$ & 0.435984 & 1.259 \\
\hline & Input clothoid $-T_{1} T_{3}$ & 0.168475 & 0.337 \\
\hline & Output clothoid $-T_{3} T_{2}$ & 0.168475 & 0.337 \\
\hline & Rectilinear segment $-T_{2} E$ & 0.475936 & 1.374 \\
\hline & $\Sigma$ & 1.248870 & 3.307 \\
\hline
\end{tabular}

The maximal time of motion (3.457s) referred to the shortest Path 1 of the length $1.2479 \mathrm{~m}$. However, the longest equalling $1.2488 \mathrm{~m}$ with the biclothoid (Path 3) was 
covered in the shortest time which was $3.307 \mathrm{~s}$. The character of dislocation courses of the trajectories being analysed for the motion along each axis was similar. Analysing the velocity courses, it was found that for Path 1 rapid changes of velocity occur in the tangent points $T_{1}$ and $T_{2}$. In the case of the other trajectories (Path 2, Path 3) these changes are also observed out they are of „mild" transitions character. Discontinuities of the course in points $T_{1}$ and $T_{2}$ are observed only for Path 1 in the acceleration diagrams. Analysing the motion along the rectilinear trajectory it can be stated, that the maximal absolute acceleration value $a_{x}$ occurs in point $T_{2}$ being $0.989 \mathrm{~m} \cdot \mathrm{s}^{-2}$. Acceleration $a_{y}$ at point $T_{l}$ reaches the maximal value of $1.244 \mathrm{~m} \cdot \mathrm{s}^{-2}$. For Path 2 , the maximal absolute value $a_{x}$ is $0.775 \mathrm{~m} \cdot \mathrm{s}^{-2}$ (point $T_{4}$ ). However, the maximal value of the component $a_{y}$ is $1.178 \mathrm{~m} \cdot \mathrm{s}^{-}$ 2 (point $T_{3}$ ). For the biclothoid (Path 3 ) the maximal absolute acceleration value $a_{x}$ is $0.604 \mathrm{~m} \cdot \mathrm{s}^{-2}$ and that of the component $a_{y}$ is $1.095 \mathrm{~m} \cdot \mathrm{s}^{-2}$

\section{CONCLUSIONS}

As follows from the numerical investigations the highest values of components of acceleration in motion along the curvilinear trajectory were found for the trajectory with the circular axis and the lowest for the trajectory with the biclothoid. Translocation, velocity and acceleration for the trajectory, in which the clothoid is use, are continuous functions in the whole range of motion. Moreover, application of polynomial function for description of acceleration causes that its course is tangent towards the time axis in the initial and final points of the trajectory. The above mentioned properties, have a significant effect on increase in positioning accuracy, reduce occurrence of dynamic loads in the manipulator kinematic system, driving energy and time of operation. The algorithm can be implemented in only few steps and therefore, it is very effective.

\section{REFERENCES}

Baur, J., Schütz, C., Pfaff, J., Buschmann, T., \& Ulbrich, H. (2014). Path Planning for a Fruit Picking Manipulator. Proceedings International Conference of Agricultural Engineering, Zurich, 06-10.07.2014.

Boryga, M. (2014). Trajectory planning of an end-effector for path with loop. Strojniški vestnik - Journal of Mechanical Engineering, 60(12), 804-814.

Boryga, M., Graboś, A., Kołodziej, P., Gołacki, K., \& Stropek, Z. (2015). Trajectory Planning with Obstacles on the Example of Tomato Harvest. Agriculture and Agricultural Science Procedia, 7, $27-34$.

Graboś, A., \& Boryga M. (2013). Trajectory planning of end-effector with intermediate point. Eksploatacja i Niezawodnosc - Maintenance and Reliability, 15(2), 182-187.

Kobryń, A. (2017). Use of Polynomial Transition Curves in the Design of Horizontal Arcs. Roads and Bridges - Drogi i Mosty, 16, 5-14.

Koc W. (2015). Identification of Transition Curves in Vehicular Roads and Railways. Logistics and Transport, 4(28), 31-42.

Sabelhaus, D., Roben, F., Helligen, L.P.M.Z., \& Lammers, P.S. (2013). Using continuous-curvature paths to generate feasible headland turn manoeuvres. Biosystems Engineering, 116(4), 399-409.

Schuetz, C., Baur, J., Pfaff, J., Buschmann, T., \& Ulbrich H. (2015). Evaluation of a Direct Optimization Method for Trajectory Planning of a 9-DOF Redundant Fruit-Picking Manipulator, Book Series: IEEE International Conference on Robotics and Automation ICRA, 2660-2666.

Wilde D.K. (2009). Computing Clothoid Segments for Trajectory Generation. The 2009 IEEE-RSJ International Conference on Intelligent Robots and Systems, 2440-2445.

Zhang, S.L., Yuan, T., Wang, D.S., Zhang, J.X., \& Li, W. (2016). Structure Optimization and Path Planning of Picking Manipulator. Proceedings of $20169^{\text {th }}$ International Symposium on Computational Intelligence and Design ISCID, Vol 2, 356-360. 